\title{
Jónsson and HS Modules over Commutative Rings
}

\author{
Greg Oman \\ Department of Mathematics, The University of Colorado, Colorado Springs, CO 80918, USA \\ Correspondence should be addressed to Greg Oman; goman@uccs.edu \\ Received 16 October 2013; Revised 14 January 2014; Accepted 20 January 2014; Published 12 March 2014 \\ Academic Editor: A. Cigdem Ozcan \\ Copyright (C) 2014 Greg Oman. This is an open access article distributed under the Creative Commons Attribution License, which \\ permits unrestricted use, distribution, and reproduction in any medium, provided the original work is properly cited. \\ Let $R$ be a commutative ring with identity and let $M$ be an infinite unitary $R$-module. (Unless indicated otherwise, all rings are \\ commutative with identity $1 \neq 0$ and all modules are unitary.) Then $M$ is called a Jónsson module provided every proper submodule \\ of $M$ has smaller cardinality than $M$. Dually, $M$ is said to be homomorphically smaller (HS for short) if $|M / N|<|M|$ for every \\ nonzero submodule $N$ of $M$. In this survey paper, we bring the reader up to speed on current research on these structures by \\ presenting the principal results on Jónsson and HS modules. We conclude the paper with several open problems.
}

\section{Introduction}

From a universal perspective, an algebra $\mathbf{A}$ is simply a set $A$ along with a collection $\mathscr{F}$ of operations on $A$, each of finite arity. (If $f \in \mathscr{F}$ has arity $n$, then $f$ is a function with domain $A^{n}$ and image contained in $A$. By convention, $A^{0}=\{\emptyset\}$. Hence the functions in $\mathscr{F}$ of arity 0 are called constants and are naturally identified with members of $A$.) The ground set $A$ is called the universe of $\mathbf{A}$. A subuniverse of an algebra $\mathbf{A}$ is a set $B \subseteq A$ which is closed under the functions in $\mathscr{F}$. In 1962, Bjarni Jónsson posed the following problem, which is now known by the moniker "Jónsson's Problem."

Problem 1 (Jósson's problem). For which infinite cardinals $\kappa$ does there exist an algebra $\mathbf{A}$ of size $\kappa$ (i.e., the ground set $A$ has size $\kappa$ ) with but finitely many operations (i.e., $\mathscr{F}$ is finite) for which every proper subuniverse $B$ of $\mathbf{A}$ has cardinality less than $\kappa$ ?

Infinite algebras $\mathbf{A}$ with finitely many operations for which every proper subuniverse of $\mathbf{A}$ has smaller cardinality than $A$ are known as Jónsson algebras. Several early but important results on these algebras were pioneered by Erdős, Rowbottom, and Silver (see [1, 2], and [3], resp.), among others.

In the modern era, the theory of Jónsson algebras has proved to be a useful tool in the investigation of large cardinals. We will not present any set-theoretic theorems on general Jónsson algebras in this paper, as that would take us too far afield. Instead, we refer the reader to Jech [4] for such results and to Coleman [5] for a less technical exposition of Jónsson algebras (which gives a treatment of Jónsson groups and rings, in particular).

We now present two natural examples of Jónsson algebras to initiate the reader.

Example 2. Let $\mathbf{A}:=(\mathbb{N},\{P\})$, where $\mathbb{N}$ is the set of natural numbers (we assume that $0 \in \mathbb{N}$ ) and $P$ is the predecessor function on $\mathbb{N}$ defined as follows:

$$
P(n):= \begin{cases}n-1, & \text { if } n>0, \\ 0, & \text { if } n=0 .\end{cases}
$$

Then $\mathbf{A}$ is a Jónsson algebra.

To see why this is true, observe that if $B$ is any subuniverse of $\mathbf{A}$ and $n \in B$; then since $B$ is closed under $P$, we conclude that $\{0,1, \ldots, n\} \subseteq B$. From this observation, it follows easily that the only infinite subuniverse of $\mathbf{A}$ is $\mathbb{N}$. Hence every proper subuniverse of $\mathbf{A}$ is finite.

Example 3. Let $p$ be a prime, and let $\mathbb{Z}\left(p^{\infty}\right)$ be the quasicyclic group $\left(\mathbb{Z}\left(p^{\infty}\right)\right.$ is the subgroup of $\mathbb{Q} / \mathbb{Z}$ consisting of the rational numbers with denominator a power of $p$ (modulo $\mathbb{Z})$ ) of type $p^{\infty}$. Then the group $\mathbb{Z}\left(p^{\infty}\right)$ is a Jónsson group (more formally, $\left(\mathbb{Z}\left(p^{\infty}\right),\{+,-, 0\}\right)$ is a Jónsson algebra, where-is the unary (additive) inverse operation on $\left.\mathbb{Z}\left(p^{\infty}\right)\right)$. 
We will not reproduce a proof of this assertion as it is well known that every proper subgroup of $\mathbb{Z}\left(p^{\infty}\right)$ has cardinality $p^{n}$ for some nonnegative integer $n$ (see pages 15-16 of Fuchs [6], e.g.). More interestingly, it can be shown that the quasicyclic groups are the only Abelian Jónsson groups; we will present a short proof of this fact (which originally appears in Scott [7]) in Section 2.

The following question now follows naturally: are there any non-Abelian Jónsson groups? O. J. Smidt posed the problem of whether there exists a countably infinite nonAbelian Jónsson group. This problem was open for roughly 50 years until, in 1980, A. Ju. Olsanskii gave such an example (Olšanskir [8]). What can be said of the existence of uncountable Jónsson groups (in ZFC)? At present, the answer is "not much." Shelah proved the existence of a Jónsson group of size $\aleph_{1}$ in [9]; for cardinals $\kappa>\aleph_{1}$, it appears to be an open question whether one can prove the existence of a Jónsson group of size $\kappa$ in ZFC. (Assuming the generalized continuum hypothesis, Shelah established the existence of a Jónsson group of size $\aleph_{a+1}$ for every ordinal $a$.)

Shelah's work was predated by related work done by McKenzie, Laffey, and Kostinsky. McKenzie proved in [10] that the only commutative Jónsson semigroups (i.e., infinite semigroups whose proper subsemigroups are all of smaller cardinality) are the quasicyclic groups $\mathbb{Z}\left(p^{\infty}\right)$. He also showed, assuming the generalized continuum hypothesis $(\mathrm{GCH})$, that every Jónsson semigroup is a group whence also a Jónsson group. A few years later, Laffey characterized the infinite rings $R$ (not assumed to be commutative or to contain an identity) for which every proper subring of $R$ is finite ([11]). It is known that every uncountable Jónsson ring is a noncommutative division ring (see Kostinsky [12]); however, it is still not known whether such rings exist.

In the early 1980s, the Jónsson property caught the attention of commutative algebraists Gilmer and Heinzer, who initiated the study of a module-theoretic analog in [13]. To wit, they define an infinite module $M$ over a $\operatorname{ring} R$ to be a Jónsson module provided $|N|<|M|$ for every proper $R$ submodule $N$ of $M$ (note that this notion is trivial if applied to a finite $R$-module $M$ since any such $M$ has the property that all proper $R$-submodules of $M$ have smaller cardinality than $M$ ). They both applied and extended their results to other algebraic structures in several subsequent papers [1418]. Various mathematicians have also contributed to the theory of Jónsson modules over the years (either by proving explicit theorems on Jónsson modules or by proving moduletheoretic results which can be easily applied to yield such theorems) including Burns et al. [19], Ecker [20], Heinzer and Lantz [21], Lawrence [22], Weakley [23], and the author of [24-26]. In Sections 2-6 of this paper, we present the principal results on Jónsson modules from their inception in the early 1980s to the present day. Our goal is to bring the reader to the forefront of research on these structures.

Sections 7-9 are devoted to the survey of a sort of dual to the class of Jónsson modules; its roots lie in a problem posed by Irving Kaplansky. To wit, in his classic text Infinite Abelian Groups, Kaplansky poses the problem of showing that $\mathbb{Z}$ is the unique infinite Abelian group $G$ with the property that $G / H$ is finite for every nonzero subgroup $H$ of $G$ (this appears as an exercise in [27]). Jensen and Miller translate this question to the realm of commutative semigroups in [28], defining an infinite commutative semigroup $S$ to be homomorphically finite (HF for short) if and only if every proper homomorphic image of $S$ is finite. They then proceed to classify all HF commutative semigroups. More generally (a priori), Ralph Tucci considers infinite commutative semigroups $S$ with the property that every proper homomorphic image of $S$ has smaller cardinality than $S$, calling such semigroups $H$-smaller (short for homomorphically smaller). He then shows that the class of $H$-smaller semigroups actually coincides with the class of HF semigroups in [29].

Several other mathematicians have considered the HF property in the context of associative rings and modules. For instance, Chew and Lawn define a ring $R$ with 1 (not assumed commutative) to be residually finite provided every proper homomorphic image of $R$ is finite. They prove various results about such rings in [30]. In [31], Levitz and Mott extend their results to rings without identity. In related work, Kearnes and the author of [32] as well as Shah [33] study the possible cardinalities of residue fields of Noetherian integral domains. (The work [32] corrects many of the results in [33] which are flawed due to an error in cardinal arithmetic (namely, that $\kappa^{\aleph_{0}}=\kappa$ whenever $\kappa$ is a cardinal of size at least $2^{\aleph_{0}}$, which is false).) Other variants of residual finiteness also appear in the literature. For example, Orzech and Ribes define an associative ring $R$ to be residually finite if and only if for every nonzero $x \in R$, there is a two-sided ideal $A$ of $R$ such that $x \notin A$ and $R / A$ is finite (see [34]). Varadarajan [35] generalizes this definition and calles an $R$-module $M$ residually finite if and only if for any $m \neq 0 \in M$, there exists a submodule $N$ of $M$ (depending on $m$ ) such that $m \notin N$ and $M / N$ is finite.

Following Tucci's terminology, Salminen and the author define an infinite module $M$ over a ring $R$ to be $H$-smaller (HS for short) provided $|M / N|<|M|$ for every nonzero submodule $N$ of $M$. Some structural results on these modules were obtained in Oman and Salminen [36] and Oman [37]. We exposit the theory of HS modules in Sections 7-9. In Section 10, we present some statements on Jónsson and HS modules which are independent of ZFC. Section 11 is devoted to listing several open problems for further research. We conclude the introduction by informing the reader that the vast majority of the ring-theoretic terminology used in this survey can be found in the text Multiplicative Ideal Theory by Robert Gilmer [38].

\section{Fundamental Results on Jónsson Modules}

As stated in Section 1, an infinite unitary module $M$ over a ring $R$ is said to be a Jónsson module provided all proper submodules of $M$ are of smaller cardinality than $M$. We have already mentioned that for any prime $p$, the quasicyclic group $\mathbb{Z}\left(p^{\infty}\right)$ is a Jónsson Abelian group, whence a Jónsson module over the ring $\mathbb{Z}$ of integers. We now present two more examples, the first of which is trivial and the second of which is a bit more exotic.

Example 4. Let $F$ be an infinite field. Then the only submodules of $F$ (as a vector space over itself) are $\{0\}$ and $F$. Thus 
$F$ is a Jónsson module over itself. More generally, if $R$ is a commutative ring and $M$ is a maximal ideal of $R$ of infinite residue, then $R / M$ is a Jónsson module over $R$.

Example 5 (see [13, Example 4.2]). Assume that $D$ is an integral domain with quotient field $K$, where $(V, M)$ is a rankone discrete valuation domain on $K$ containing $D$ (here, $M$ is the maximal ideal of $V)$ and $V / M \cong D / P$ is a finite field, where $P$ is the center of $V$ on $D$ (i.e., $P:=M \cap D$ ). Then $K / V$ is a Jónsson module over $D$.

Finally, we are ready to discuss the theory of Jónsson modules over a commutative ring. We begin with the following simple lemma.

Lemma 6. Every Jónsson module is indecomposable.

Proof. Suppose that $M$ is a Jónsson module over the $\operatorname{ring} R$ and that $M=A \oplus B$ for some $R$-submodules $A$ and $B$ of $M$. Since $M$ is infinite, it follows from basic cardinal arithmetic that $|A|=|M|$ or $|B|=|M|$. Since $M$ is Jónsson, we conclude that either $A=M$ or $B=M$, and the proof is complete.

Remark 7. Example 4 and Lemma 6 imply that if $F$ is a field and $V$ is an $F$-vector space, then $V$ is a Jónsson module over $F$ if and only if $F$ is infinite and $V \cong_{F} F$.

We now establish a fundamental result due to Gilmer and Heinzer.

Proposition 8 (see [13, Proposition 2.5]). Let $M$ be a Jónsson module over the ring $R$.

(1) For all $r \in R$, either $r M=M$ or $r M=\{0\}$.

(2) $\operatorname{Ann}_{R}(M):=\{r \in R: r M=\{0\}\}$ is a prime ideal of $R$.

Proof. Assume that $M$ is a Jónsson module over the ring $R$.

(1) Let $r \in R$ be arbitrary. If $|r M|=|M|$, then since $M$ is Jónsson; we deduce that $r M=M$, and we are done. Thus assume that $|r M|<|M|$. Let $\varphi: M \rightarrow r M$ be the natural map, and let $K$ be its kernel. Then $M / K \cong_{R} r M$. Thus $|M / K|=|r M|<|M|$. Since $|M|=$ $|M / K| \cdot|K|$ and since $|M / K|<|M|$, it follows from basic cardinal arithmetic that $|K|=|M|$. Again, since $M$ is Jónsson, we see that $K=M$. But this implies that $r M=\{0\}$, and the proof of (1) is complete.

(2) Suppose that $r, s \in R-\operatorname{Ann}_{R}(M)$. Then by (1), $r M=$ $M$ and $s M=M$. Hence $r s M=r M=M$, and $r s \notin$ $\operatorname{Ann}_{R}(M)$. This concludes the proof.

By (2) of Proposition 8 (modding out the annihilator), there is no loss of generality in restricting our study of Jónsson modules to faithful modules over a domain. Remark 7 implies that we may restrict even further to faithful modules over domains which are not fields. The following corollaries now follow easily.
Corollary 9 (see [13, Proposition 2.2]). Let $R$ be an infinite ring. Then $R$ is a field if and only if $R$ is Jónsson as a module over itself.

Proof. We saw in Example 4 that every infinite field is Jónsson as a module over itself. Conversely, assume that $R$ is an infinite ring which is Jónsson as a module over itself. We will show that $R$ is a field. Since $R$ has an identity, it is clear that $\operatorname{Ann}_{R}(R)=\{0\}$. But now (1) of Proposition 8 implies that $r R=R$ for every nonzero $r \in R$, whence $R$ is a field.

Corollary 10 (see [26, Corollary 1]). There are no Jónsson modules over a finite ring.

Proof. Let $R$ be a finite ring, and suppose by way of contradiction that $M$ is a Jónsson $R$-module. By (2) of Proposition 8 , $P:=\operatorname{Ann}_{R}(M)$ is a prime ideal of $R$. But since $R$ is finite, $R / P$ is a field. Thus $M$ is naturally a Jónsson vector space over the finite field $R / P$. But since $M$ is infinite, $M$ is an infinite direct sum of copies of $R / P$, contradicting Lemma 6 .

As promised in the introduction, we now present a simple proof of Scott's result that the quasicyclic groups are the only Abelian Jónsson groups.

Proposition 11 (see [7, Remark 1, page 196]). The only Abelian Jónsson groups are the quasicyclic groups $\mathbb{Z}\left(p^{\infty}\right)$, p a prime.

Proof. As noted in Example 3, every quasicyclic group $\mathbb{Z}\left(p^{\infty}\right)$ is a Jónsson Abelian group. Conversely, suppose that $G$ is a Jónsson Abelian group. We will prove that $G \cong \mathbb{Z}\left(p^{\infty}\right)$ for some prime $p$. By (2) of Proposition 8, we have that $\operatorname{Ann}_{\mathbb{Z}}(G)$ is a prime ideal of $\mathbb{Z}$. Thus $\operatorname{Ann}_{\mathbb{Z}}(G)=\mathbb{Z} p$ for some prime $p$ or $\operatorname{Ann}_{\mathbb{Z}}(G)=\{0\}$. Corollary 10 precludes the former case from being a possibility, whence $G$ is a faithful $\mathbb{Z}$-module. But now (1) of Proposition 8 implies that $G$ is a divisible Abelian group. The structure theorem for divisible Abelian groups yields that $G$ is isomorphic to a direct sum of copies of $\mathbb{Q}$ and $\mathbb{Z}\left(p^{\infty}\right)$ for various primes $p$. We invoke Lemma 6 to conclude that $G \cong \mathbb{Q}$ or $G \cong \mathbb{Z}\left(p^{\infty}\right)$ for some prime $p$. Since $\mathbb{Q}$ is clearly not a Jónsson group ( $\mathbb{Z}$ is a proper infinite subgroup), we deduce that $G \cong \mathbb{Z}\left(p^{\infty}\right)$ for some prime $p$, completing the proof.

The previous proposition raises several natural questions, and we will deal with them systematically in the next few sections. We conclude the section by answering one such query. As noted after the proof of Proposition 8, we may restrict our study of Jónsson modules to faithful modules over a domain $D$ which is not a field. The previous proposition shows that $\mathbb{Z}$ admits a faithful Jónsson module. Hence we ask the following.

Question 1. Does every domain $D$ which is not a field admit a faithful Jónsson module?

The answer is "no." More specifically, consider the following. 
Proposition 12 (see [24, Theorems 7-9]). The following domains do not admit faithful Jónsson modules:

(1) the polynomial ring $F\left[x_{1}, x_{2}, \ldots, x_{n}\right]$, where $F$ is an infinite field,

(2) the power series ring $F\left[\left[t_{1}, t_{2}, \ldots, t_{n}\right]\right]$, where $F$ is a field and either $F$ is infinite or $n>1$,

(3) valuation domains of positive (finite) Krull dimension.

\section{Countable Jónsson Modules}

Recall from Proposition 11 that the only Jónsson $\mathbb{Z}$-modules are the (countable) quasicyclic groups $\mathbb{Z}\left(p^{\infty}\right)$, where $p$ is a prime number. Thus the following question arises naturally.

Question 2. Is it possible to characterize the countable Jónsson modules over an arbitrary ring?

We will show that the answer to this question is "yes." The solution follows from essentially "gluing together" results of Gilmer, Heinzer, Lantz, and Weakley. We begin with a result of Gilmer and Heinzer.

Lemma 13 (see [38, (2) of Theorem 3.1]). Suppose that $M$ is a countable faithful Jónsson module over a domain $D$ which is not a field. Then D possesses a maximal ideal J of finite index in $D$.

We now introduce some terminology of which we will shortly make use. Heinzer and Lantz [21] and Weakley [23] call a module $M$ over a ring $R$ almost finitely generated if $M$ is not finitely generated, but all proper submodules of $M$ are finitely generated. A domain $D$ is said to be an almost DVR provided the integral closure $\bar{D}$ of $D$ (in the quotient field $K$ of $D$ ) is a DVR which is finitely generated as a $D$-module. Finally, $R$-modules $M$ and $N$ are said to be quotient equivalent (denoted $M \sim_{e} N$ ) if each module is a homomorphic image of the other (this terminology is due to Eben Matlis).

We recall the following results of Heinzer, Lantz, and Weakley, and then we characterize the countable Jónsson modules.

Lemma 14 (see [21, Proposition 2.2]). Let $D$ be a domain with quotient field $K$ and suppose that $(S, J)$ is an almost DVR between $D$ and $K$ (here $J$ is the maximal ideal of $S$ ). Then $K / S$ is an almost finitely generated $D$-module if $S / J$ is a D-module of finite length.

Lemma 15 (see [23, Proposition 2.2]). Let $M$ be an Artinian almost finitely generated module over the ring $R$, and let $q:=$ $\operatorname{Ann}_{R}(M)$ ( $q$ must be a prime ideal of $R$ ). Then there is a discrete valuation ring $V$ between $R / q$ and $Q(R / q)$ (the quotient field of $R / q)$ such that $M \sim_{e} Q(R / q) / V$.

Theorem 16 (see [25, Theorem 2]). Let $D$ be an infinite domain with quotient field $K$, and suppose that $M$ is a countably infinite D-module. Then $M$ is a faithful Jónsson module if and only if one of the following holds.

(1) $D$ is a field and $M \cong_{D} D$.
(2) There is a discrete valuation overring $V$ of $D$ with finite residue field such that $M \cong{ }_{D} K / N$, where $N$ is a proper $D$-submodule of $K$ containing $V$.

Proof. Assume that $D$ is an infinite domain with quotient field $K$ and that $M$ is a countably infinite $D$-module. If $D$ is a field and $M \cong_{D} D$, then $M$ is trivially a faithful Jónsson $D$-module. Suppose now that $V$ is a discrete valuation overring of $D$ with a finite residue field and that $M \cong_{D} K / N$, where $N$ is a proper $D$-submodule of $K$ containing $V$. Let $(v)$ be the maximal ideal of $V$. It is easy to show that every cyclic $V$-submodule of $K / V$ is finite. It now follows trivially that every cyclic $D$-submodule of $K / V$ is finite. Since $V /(v)$ is finite, clearly $V /(v)$ has finite length as a $D$-module. Lemma 14 implies that $K / V$ is an almost finitely generated $D$-module. This fact along with the fact that every cyclic $D$-submodule of $K / V$ is finite implies that $K / V$ is a (faithful) Jónsson $D$-module. Recall that $M \cong_{D} K / N \cong_{D}(K / V) /(N / V)$, whence $M$ is a homomorphic image of the countable Jónsson $D$-module $K / V$. It follows that $M$ is a faithful Jónsson $D$-module as well.

Now assume that $M$ is an arbitrary countable faithful Jónsson module over $D$. If $M$ is finitely generated, then Corollary 2.3 of [13] shows that $D$ is a field and $M \simeq_{D} D$. Thus we suppose that $M$ is infinitely generated over $D$. Since $M$ is Jónsson and countably infinite, every proper $D$-submodule of $M$ is finite. Hence $M$ is an almost finitely generated Artinian $D$-module. Lemma 15 applies, and we deduce that there is a discrete valuation overring $V$ of $D$ such that $M \sim_{e} K / V$. Hence $M \cong{ }_{D} K / N$ for some proper $D$-submodule $N$ of $K$ containing $V$. Further, $K / V$ is a homomorphic image of $M$, whence is a Jónsson $D$-module. Thus $K / V$ is also a Jónsson $V$-module. Lemma 13 implies that $V$ has a finite residue field, and the proof is complete.

Having classified the countable Jónsson modules, it is natural to investigate the following query.

Question 3. For which domains $D$ (which are not fields) is every faithful Jónsson $D$-module countable?

Recall from Proposition 11 that the ring $\mathbb{Z}$ of integers possesses the above property. More generally, we have the following.

Proposition 17 (see [25, Theorem 3]). Suppose that D is a one-dimensional Noetherian domain. Then every faithful Jónsson D-module is countable.

Before stating our next result, we remind the reader that the generalized continuum hypothesis $(\mathrm{GCH})$ is that statement that for every infinite cardinal number $\kappa$, there are no cardinals strictly between $\kappa$ and $2^{\kappa}$. It is well known that GCH can neither be proved nor refuted from the axioms of ZFC (assuming ZFC is consistent); these results are due to Kurt Gödel and Paul Cohen.

What can be said of the cardinality of faithful Jónsson modules over a general Noetherian domain $D$ (which is not a field)? Here is a partial answer. 
Proposition 18 (see [25, Theorem 4]). Assume that GCH holds. Then if D is a Noetherian domain which is not a field and $M$ is a faithful Jónsson module over $D$, then $M$ is countable.

Thus one cannot prove the existence of uncountable faithful Jónsson modules over a Noetherian domain $D$ (which is not a field) in ZFC unless ZFC is inconsistent. (If one could do so, then one would have a proof in $\mathrm{ZFC}$ that $\mathrm{GCH}$ fails. By Gödel's work [39], this is only possible if ZFC is inconsistent.) It remains open whether the nonexistence of uncountable faithful Jónsson modules over a Noetherian domain $D$ (which is not a field) can be proved in ZFC.

\section{Uncountable Jónsson Modules}

Having characterized the countable Jónsson modules in the previous section, we consider the following question.

Question 4. Is it possible to classify the uncountable Jónsson modules over an arbitrary ring?

At present, answering Question 4 seems untenable. Recall from Section 1 that it is not even known if one can prove the existence of a Jónsson group of cardinality $\aleph_{2}$ in ZFC. Though we cannot answer Question 4 at this time, we sketch a proof that for every uncountable cardinal $\kappa$, there exists a domain $D$ which is not a field and a faithful Jónsson $D$-module $M$ of cardinality $\kappa$.

Proposition 19 (see [36, Corollary 5.4]). Let $\kappa$ be an uncountable cardinal. There exists a domain $D$ which is not a field and a faithful Jónsson D-module of cardinality $\kappa$.

Proof. Let $\kappa$ be an uncountable cardinal, and consider the additive group $G$ defined by $G:=\bigoplus_{\kappa} \mathbb{Z}$ (thus $G$ is simply the direct sum of $\kappa$ copies of $\mathbb{Z}$ ). Now equip $G$ with the reverse lexicographic order (the details follow). A nonzero element $\left(g_{i}\right) \in G$ has but finitely many nonzero entries. Let $j$ be greatest such that $g_{j} \neq 0$. If $g_{j}>0$, then we say that $\left(g_{i}\right)$ is positive. Now let $P$ be the set of positive elements of $G$ (i.e., the positive cone of the order). Then one checks easily that $P$ is closed under addition and that $\{P,\{0\},-P\}$ forms a partition of $G$. Hence the order $<$ defined on $G$ by $x<y$ if and only if $y-x \in P$ is a translation-invariant total order. It is easy to show that for every $g>0$, the interval $[0, g]:=\{x \in G: 0 \leq$ $x \leq g$ \} has cardinality strictly less than $\kappa$.

Now let $S$ denote the submonoid of $G$ consisting of the nonnegative elements of $G$, and let $D:=\mathbb{F}_{2}[S]$ denote the semigroup ring of $S$ over the field $\mathbb{F}_{2}$ of two elements. Every nonzero element of $D$ may be written uniquely in the form

$$
x^{g_{1}}+x^{g_{2}}+\cdots+x^{g_{n}}
$$

where $g_{1}<g_{2}<\cdots<g_{n}$. Now define a map $v: D \rightarrow$ $G \cup\{\infty\}$ by $v\left(x^{g_{1}}+x^{g_{2}}+\cdots+x^{g_{n}}\right):=g_{1}$, and $v(0):=\infty$ (recall that $g+\infty=\infty+g=\infty+\infty=\infty$ and $g<\infty$ for every $g \in G$ ). It follows (from Proposition 18.1 of [38], e.g.) that $v$ may be extended to a valuation on the quotient field $K$ of $D$ by defining $v(a / b):=v(a)-v(b)$. Let $V$ be the valuation ring of $v$. Then one proves that $V$ is not a field, $|V|=|K / V|=\kappa$, and $K / V$ is a faithful Jónsson module over $V$.

\section{Large Jónsson Modules}

Having considered both small (countable) and large (of arbitrary uncountable cardinality) Jónsson modules, we change gears and study a sort of "relative largeness." Let us say that an infinite $R$-module $M$ is large if its cardinality exceeds that of $R$, that is, if $|M|>|R|$. Note that from Proposition $11, \mathbb{Z}$ does not admit any large Jónsson modules. This observation leads to the following question.

Question 5. Does there exist a ring $R$ and Jónsson $R$-module $M$ such that $|M|>|R|$ ?

This question is open in general, though there are partial answers in the literature which we will exposit shortly. We begin with a lemma and then recall some definitions from basic set theory.

Lemma 20 (see [26, Theorem 2.1]). Let $R$ be a ring, and suppose that $M$ is a Jónsson module over $R$. Then either $R$ is a field and $M \cong_{R} R$, or $M$ is a torsion module (i.e., for every $m \in M$, there exists some nonzero $r \in R$ such that $r m=0$ ).

Now let $\kappa$ be an infinite cardinal. The cofinality of $\kappa$, denoted $c f(\kappa)$, is the least cardinal $\lambda$ such that $\kappa$ is the sum of $\lambda$ many cardinals, each smaller than $\kappa$. The cardinal $\kappa$ is called regular if $c f(\kappa)=\kappa$ and singular if $c f(\kappa)<\kappa$. The regular cardinals include $\aleph_{0}$ as well as every successor cardinal (Often, a successor cardinal is denoted by $\lambda^{+}$, which is simply the smallest cardinal larger than the cardinal $\lambda$.), that is, every cardinal of the form $\aleph_{a+1}$ for some ordinal $a$.

Though Question 5 is open, we can show that if $M$ is a Jónsson module over a ring $R$, then the cofinality of $|M|$ cannot exceed $|R|$.

Proposition 21 (see [26, Proposition 3]). Let $R$ be a ring and $M$ be a Jónsson R-module. Then $c f(|M|) \leq|R|$.

Proof. Suppose by way of contradiction that $R$ is a ring and $M$ is a Jónsson $R$-module such that $|R|<c f(|M|)$. Let $P:=$ $\operatorname{Ann}_{R}(M)$. Then $|R / P| \leq|R|<c f(|M|) \leq|M|$, whence $|R / P|<|M|$. Lemma 20 implies that $M$ is a (faithful) torsion module over the domain $D:=R / P$. Since $M$ is a faithful Jónsson $D$-module, it follows that for every $d \in D-\{0\}$, the module $M[d]:=\{m \in M: d m=0\}$ has smaller cardinality than $M$. However, as $M$ is torsion, we have

$$
M=\bigcup_{d \in D-\{0\}} M[d]
$$

But now we have expressed $M$ as the union of at most $|D|$ many subsets, each of smaller cardinality than $|M|$. Hence $c f(|M|) \leq|D| \leq|R|$, a contradiction.

Corollary 22 (see [26, Corollary 3]). Let $R$ be a ring. Then $R$ does not admit a large Jónsson module of regular cardinality. 
Proof. If $M$ is a Jónsson module over $R$ of regular cardinality, then, by the previous proposition, $|M|=c f(M) \leq|R|$.

We now pause to showcase the utility of the previous corollary with an application. We first remind the reader that a module $M$ over a ring $R$ is uniserial provided the $R$ submodules of $M$ form a chain with respect to set inclusion.

Proposition 23. Let $R$ be a ring. Then $R$ admits neither $a$ large Artinian module (The nonexistence of a large Artinian module follows from a more general result due to Dan Anderson [40]: Artinian modules over commutative rings are countably generated.) nor a large uniserial module.

Proof. Let $R$ be a ring. We first show that $R$ does not admit a large Artinian $R$-module. Suppose by way of contradiction that $M$ is a large Artinian module over $R$. Assume first that $R$ is finite. Since $M$ is Artinian, there exists an $R$-submodule $N$ of $M$ which is minimal with respect to being countably infinite. But then $N$ is a Jónsson module over the finite ring $R$, contradicting Corollary 10. Thus $R$ is infinite. Now let $N$ be an $R$-submodule of $M$ which is minimal with respect to having cardinality $|R|^{+}$. But then $N$ is a large Jónsson $R$-module of regular cardinality, contradicting Corollary 22.

We now show that $R$ does not admit a large uniserial $R$ module. Suppose by way of contradiction that $M$ is a large uniserial $R$-module. Let $N$ be an arbitrary proper submodule of $M$, and let $m \in M-N$. Then since $M$ is uniserial, we have $N \subseteq R m$. Hence $|N| \leq|R|$. Since $M$ is large, it follows that $M$ is a Jónsson module over $R$. Corollary 10 applies again, and we conclude that $R$ is infinite. But now choose an arbitrary submodule $N$ of $M$ of cardinality $|R|^{+}$. Then $N$ is uniserial, and by what we just proved, $N$ is Jónsson over $R$. But then $N$ is a large Jónsson $R$-module of regular cardinality, contradicting Corollary 22.

We conclude this section by showing that one cannot prove the existence of large Jónsson modules in ZFC; it remains open whether one can disprove the existence of large Jónsson modules in ZFC. We first recall the following definition.

Definition 24. Let $R$ be a ring and $M$ an $R$-module, and suppose that $I \subseteq M$. Then $I$ is independent provided $I$ generates a direct sum in $M$, that is, if

$$
\langle I\rangle=\bigoplus_{m \in I} R m
$$

We will utilize the following result of Andreas Ecker which gives some relationships between $|R|,|M|$, and $|I|$.

Lemma 25 (see [20]). Suppose that $R$ is an infinite ring and that $I$ is a maximal independent set in an $R$-module $M$ (which exists by Zorn's Lemma). Then the following hold.

(1) If $I=\emptyset$, then $M=\{0\}$.

(2) If $|I|=1$, then $|M| \leq 2^{|R|}$.

(3) If $|I|>1$, then $|M| \leq|I|^{|R|}$.
Proposition 26 (see [26, Corollary 4]). Assume that GCH holds. Then large Jónsson modules do not exist.

Proof. Suppose GCH holds and assume by way of contradiction that $R$ is a ring and $M$ is a Jónsson $R$-module such that $|M|>|R|$. Further, let $I$ be a maximal independent set in $M$. Corollary 50 implies that $R$ is infinite. Part (1) of Lemma 25 yields that $I \neq \emptyset$. If $I$ is a singleton, then we deduce from (2) of Lemma 25 and $\mathrm{GCH}$ that $|M|=|R|^{+}$. But then $M$ is a large Jónsson $R$-module of regular cardinality, contradicting Corollary 22. Thus we conclude that $|I|>1$. One now applies (3) of Lemma 25 and GCH to prove that $|I|=|M|$. But then

$$
\left|\bigoplus_{m \in I} R m\right|=|M| \text {. }
$$

Since $M$ is a Jónsson module, it follows that $\bigoplus_{m \in I} R m=M$, contradicting that $M$ is indecomposable (Lemma 6).

Remark 27. The nonexistence of large Jónsson modules over Noetherian rings can be proved in ZFC. This follows immediately from the following well-known result (which can be found in $[19,22]$, e.g.): let $R$ be a Noetherian ring, and suppose that $M$ is a large $R$-module. Then $M$ possesses an independent set $I$ of the same cardinality as $M$.

\section{Strongly Jónsson Modules}

Note that if $M$ is a Jónsson module over the $\operatorname{ring} R$, then $|N| \neq|M|$ for every proper $R$-module $N$ of $M$. We now strengthen the definition of "Jónsson module" as follows.

Definition 28. Let $R$ be a ring and let $M$ be an $R$-module (unlike the definition of "Jónsson module," we allow $M$ to be finite). Then we call $M$ strongly Jónsson provided $|K| \neq|N|$ for any two distinct $R$-submodules $K$ and $N$ of $M$.

The terminology is justified since an infinite strongly Jónsson $R$-module $M$ is clearly also a Jónsson $R$-module. As in Section 2, we classify the strongly Jónsson Abelian groups as a jumping-off point.

Proposition 29. Let $G$ be an Abelian group. Then $G$ is strongly Jónsson (i.e., $G$ is a strongly Jónsson $\mathbb{Z}$-module) if and only if $G \cong \mathbb{Z}\left(p^{\infty}\right)$ for some prime $p$ or $G \cong \mathbb{Z} /(n)$ for some positive integer $n$.

Proof. It is known that for any prime $p$, every proper subgroup of $\mathbb{Z}\left(p^{\infty}\right)$ is finite of order $p^{n}$ for some nonnegative integer $n$. Moreover, for every nonnegative integer $n, \mathbb{Z}\left(p^{\infty}\right)$ possesses a unique subgroup of cardinality $p^{n}$ (see Fuchs [41], pages $23-25)$. It follows that distinct subgroups of $\mathbb{Z}\left(p^{\infty}\right)$ have distinct cardinalities. It is also well known that $\mathbb{Z} /(n)$ enjoys this property for every positive integer $n$ (see Hungerford [42], page 37$)$. Thus $\mathbb{Z}\left(p^{\infty}\right)$ and $\mathbb{Z} /(n)$ are strongly Jónsson Abelian groups.

Conversely, suppose that $G$ is a strongly Jónsson Abelian group. If $G$ is infinite, then $G$ is an Abelian Jónsson group, whence $G \cong \mathbb{Z}\left(p^{\infty}\right)$ for some prime $p$ by Proposition 11 . Now assume that $G$ is finite. Then, by the fundamental theorem of 
finitely generated Abelian groups, $G$ is a finite direct sum of cyclic groups of prime power order. Clearly, no two distinct summands can have cardinality a power of the same prime $p$, lest $G$ possess two distinct subgroups of order $p$. We conclude that $G \cong \mathbb{Z} /(n)$ for some positive integer $n$.

Employing a classical theorem of Baer, we can show that the conclusion of the previous proposition holds even without assuming that $G$ is Abelian.

Proposition 30. Let $G$ be a group with the property that distinct subgroups of $G$ have distinct cardinalities. Then $G$ is Abelian.

Proof. Assume that distinct subgroups of $G$ have distinct cardinalities, and suppose by way of contradiction that $G$ is nonAbelian. We first claim that every subgroup of $G$ is normal. Indeed, let $H<G$, and let $g \in G$ be arbitrary. Then clearly $|H|=\left|g \mathrm{Hg}^{-1}\right|$, whence by the condition on $G, H=g \mathrm{Hg}^{-1}$, and $H$ is normal. Hence $G$ is a Hamiltonian group (i.e., $G$ is non-Abelian and all subgroups of $G$ are normal). An old result of Baer (Baer [43]) yields that $G \cong Q_{8} \times P$, for some torsion Abelian group $P$ that has no elements of order $4\left(Q_{8}\right.$ denotes the quaternion group of order 8). But then we are forced to conclude that $Q_{8}$ inherits the property that distinct subgroups have distinct cardinalities. However, $Q_{8}$ has three subgroups of order 4 , a contradiction.

Having classified the strongly Jónsson groups, we devote the remainder of this section to classifying the strongly Jónsson modules over an arbitrary ring. Describing the infinite strongly Jónsson modules is fairly straightforward; it is the classification of the finite strongly Jónsson modules that requires some work. Let us agree to call a ring $R$ which is strongly Jónsson as a module over itself a strongly Jónsson ring (this is, of course, equivalent to the assertion that distinct ideals of $R$ have distinct cardinalities). It follows immediately from Proposition 29 that the direct sum of the groups $\mathbb{Z} /(n)$ and $\mathbb{Z} /(m)$ is strongly Jónsson (as a $\mathbb{Z}$-module) if and only if $m$ and $n$ are relatively prime. A natural question is the following: when is a direct product of finite strongly Jónsson rings strongly Jónsson? It is possible for the direct product of two strongly Jónsson rings to be strongly Jónsson even if the rings are powers of the same prime, as the ring $R:=$ $\mathbb{F}_{p} \times \mathbb{F}_{p^{2}}$ witnesses. On the other hand, $S:=\mathbb{F}_{p} \times \mathbb{F}_{p^{2}} \times \mathbb{F}_{p^{3}}$ has two distinct ideals of cardinality $p^{3}$ whence is not strongly Jónsson. We now work toward answering this question (and toward a complete classification of the strongly Jónsson modules, more generally). We begin with a definition.

Definition 31 (Gilmer [38], page 8). Let $R$ be a ring, and let $M$ be an Abelian group which is a left module over both the rings $R$ and $S$. Say that the structure of $M$ as an $R$-module is essentially the same as the structure of $M$ as an $S$-module if and only if $R m=S m$ for every $m \in M$.

It is easy to see that if the structure of $M$ as an $R$-module is essentially the same as the structure of $M$ as an $S$-module, then the set of $R$-submodules of $M$ is the same as the set of
$S$-submodules of $M$. We illustrate this concept with a natural example.

Example 32. Let $R$ be a ring, $M$ an $R$-module, and $\operatorname{Ann}_{R}(M)$ the annihilator of $M$ in $R$. Then the structure of $M$ as an $R$ module is essentially the same as the structure of $M$ as an $R / \operatorname{Ann}_{R}(M)$-module.

We now introduce two more definitions, a lemma, and two examples before presenting our classification of the strongly Jónsson modules.

Let $S$ be a commutative semigroup, and let $S_{1}, S_{2}, \ldots, S_{k}$ be subsets of $S$. Then the product set $S_{1} S_{2} \cdots S_{k}$ is defined by $S_{1} S_{2} \cdots S_{k}:=\left\{s_{1} s_{2} \cdots s_{k}: s_{i} \in S_{i}\right.$ for $\left.1 \leq i \leq k\right\}$. Suppose further that each $S_{i}$ is a finite set. Let us say that the collection $\left\{S_{1}, S_{2}, \ldots, S_{k}\right\}$ is product-maximal provided $S_{1} S_{2} \cdots S_{k}$ is as large as possible, that is, if $\left|S_{1} S_{2} \cdots S_{k}\right|=\left|S_{1} \times S_{2} \times \cdots \times S_{k}\right|$. The following simple lemma gives a useful characterization of the product-maximal property.

Lemma 33 (see [37], Lemma 6). Let $S$ be a commutative semigroup, and let $S_{1}, S_{2}, \ldots, S_{k}$ be finite subsets of $S$. Then $\left\{S_{1}, S_{2} \ldots, S_{k}\right\}$ is product-maximal if and only if the following property holds.

(P) If $x_{1} x_{2} \cdots x_{k}=y_{1} y_{2} \cdots y_{k}$ with each $x_{i}, y_{i} \in S_{i}$, then $x_{i}=y_{i}$ for all $i, 1 \leq i \leq k$.

Proof. Assume that $S$ is a commutative semigroup and that $S_{1}, S_{2} \ldots, S_{k}$ are finite subsets of $S$. Suppose first that $\left\{S_{1}, S_{2} \ldots, S_{k}\right\}$ is product-maximal. We will verify property (P). Define $\varphi: S_{1} \times S_{2} \cdots \times S_{k} \rightarrow S_{1} S_{2} \cdots S_{k}$ by $\varphi\left(s_{1}, s_{2} \ldots, s_{k}\right):=s_{1} s_{2} \cdots s_{k}$. Clearly $\varphi$ is onto. Since $\left\{S_{1}, S_{2} \ldots, S_{k}\right\}$ is product-maximal, it follows that $\varphi$ is a surjective map between two finite sets of the same cardinality. We conclude that $\varphi$ is one-to-one. Property (P) now follows. We omit the easy proof of the converse.

Our interest in the previous lemma will be in the context of the semigroup $\left(\mathbb{Z}^{+}, \cdot\right)$ of positive integers under multiplication. In this setting, the reader may notice that our property (P) above is somewhat related to the following well-studied concept (due to Erdős) in additive number theory: a subset $S \subseteq \mathbb{Z}^{+}$is a multiplicative Sidon set if and only if $a b=c d$ implies that $\{a, b\}=\{c, d\}$ for all $a, b, c, d \in S$. We now give two easy examples illustrating the product-maximal property.

Example 34. Let $S_{1}=\{2,3\}$ and $S_{2}=\{4,6\}$. Then $\left\{S_{1}, S_{2}\right\}$ is not product-maximal since $\left|S_{1} S_{2}\right|=3 \neq 4=\left|S_{1} \times S_{2}\right|$.

Example 35. If $S_{1}, S_{2}, \ldots S_{k}$ are pairwise relatively prime finite sets of positive integers (i.e., if $i \neq j$ and $x \in S_{i}, y \in S_{j}$, then $x$ and $y$ are relatively prime), then $\left\{S_{1}, S_{2}, \ldots, S_{k}\right\}$ is productmaximal.

We are almost ready to classify the strongly Jónsson modules. For the purposes of the next theorem, we define the following: if $R$ is a ring, then we let $\mathscr{C}(R):=\{|I|: I$ is an ideal of $R$ \} (thus $\mathscr{C}(R)$ is simply the set of cardinal numbers of the ideals of $R$ ). 
Theorem 36 (see [37, Theorem 1]). Let $R$ be a ring, and let $M$ be a nonzero $R$-module. Then $M$ is a strongly Jónsson $R-$ module if and only if one of the following holds.

(I) There exist discrete valuation rings $\left(V_{1}, m_{1}\right),\left(V_{2}, m_{2}\right), \ldots,\left(V_{n}, m_{n}\right)$, each with finite residue fields and positive integers $k_{1}, k_{2}, \ldots, k_{n}$, such that if the ring $S:=V_{1} /\left(m_{1}^{k_{1}}\right) \times V_{2} /\left(m_{2}^{k_{2}}\right) \times \cdots \times V_{n} /\left(m_{n}^{k_{n}}\right)$, then

(a) $M \cong_{R} S$. Moreover, the structure of $S$ as an $R$ module is essentially the same as the structure of $S$ as an $S$-module,

(b) $\left\{\mathscr{C}\left(V_{1} /\left(m_{1}^{k_{1}}\right)\right), \mathscr{C}\left(V_{2} /\left(m_{2}^{k_{2}}\right)\right) \ldots, \mathscr{C}\left(V_{n} /\left(m_{n}^{k_{n}}\right)\right)\right\}$ is product-maximal in the semigroup $\left(\mathbb{Z}^{+}, \cdot\right)$.

(II) $\operatorname{End}_{R}(M)$ (the endomorphism ring of $\left.M\right):=(V, m)$ is a complete discrete valuation ring with a finite residue field, and the structure of $M$ as an R-module is essentially the same as the structure of $M$ as a $V$-module. Moreover, if $K$ is the quotient field of $V$, then $M \cong_{V} K / V$.

(III) There exists a maximal ideal $J$ of $R$ such that $M \cong_{R} R / J$.

\section{Fundamental Results on HS Modules}

Having surveyed the important results on Jónsson modules, we now change gears and investigate a sort of dual notion. Recall from the Introduction that an infinite module $M$ over a ring $R$ is called homomorphically smaller (HS for short) provided $|M / N|<|M|$ for every nonzero submodule $N$ of $M$. As in Section 2, we begin with several examples.

Example 37. Let $F$ be a field and let $M$ be an infinite $F$-vector space. Then $M$ is HS if and only if $F$ is infinite and $M \simeq_{F} F$.

In light of this example, we may restrict ourselves to the study of HS modules over a ring $R$, that is, not a field without loss of generality.

Example 38. The ring $\mathbb{Z}$ of integers is $\mathrm{HS}$ as a module over itself.

Example 39. If $F$ is a finite field, then both the polynomial ring $F[x]$ and the power series ring $F[[t]]$ are HS as modules over themselves.

Example 40 (see [36, Theorem 2.8]). The valuation ring $V$ constructed in Proposition 19 is HS as a module over itself.

We now commence the building of the theory of HS modules with the following proposition (compare to Proposition 8).

Proposition 41 (see [36, (iv) of Proposition 3.2]). Let $R$ be a ring, and let $M$ be an HS R-module. Then $\operatorname{Ann}_{R}(M)$ is a prime ideal of $R$.

Proof. Assume that $R$ is a ring and that $M$ is an HS $R$ module. Let $r, s \in R$ and suppose that $r \notin \operatorname{Ann}_{R}(M)$ and $s \notin \operatorname{Ann}_{R}(M)$. We will prove that $r s \notin \operatorname{Ann}_{R}(M)$. Toward this end, observe first that $M / s M \cong_{R}(M / r s M) /(s M / r s M)$. It follows that

$$
\left|\frac{M}{s M}\right| \cdot\left|\frac{s M}{r s M}\right|=\left|\frac{M}{r s M}\right| .
$$

Note that the map $\varphi: M / r M \rightarrow s M / r s M$ defined by $r M+$ $m \mapsto r s M+s m$ is well-defined and onto. Thus $|s M / r s M| \leq$ $|M / r M|$. We conclude from (6) above that

$$
\left|\frac{M}{r s M}\right|=\left|\frac{M}{s M}\right| \cdot\left|\frac{s M}{r s M}\right| \leq\left|\frac{M}{s M}\right| \cdot\left|\frac{M}{r M}\right| .
$$

Since $r \notin \operatorname{Ann}_{R}(M)$ and $s \notin \operatorname{Ann}_{R}(M)$, we see that $r M \neq\{0\} \neq s M$. As $M$ is HS, we deduce that $|M / r M|<|M|$ and $|M / s M|<|M|$. Thus by (7), we get $|M / r s M| \leq|M / s M|$. $|M / r M|<|M|$. Since $|M / r s M|<|M|$, it is clear that $r s \notin$ $\operatorname{Ann}_{R}(M)$, and the proof is complete.

Thus by Example 37 and Proposition 41, there is no loss of generality in restricting our study of HS modules to faithful modules over domains which are not fields.

We conclude this section with a structure theorem for $\mathrm{HS}$ modules.

Theorem 42 (see [36, Theorem 3.3]). Let $D$ be a domain (which is not a field) with quotient field $K$ and let $M$ be a faithful module over D. Consider the following conditions.

(a) D is HS as a module over itself.

(b) $D \subseteq M \subseteq K$ (up to D-module isomorphism).

(c) There is a generating set $S$ for $M$ over $D$ with $|S|<|D|$.

(d) $|M / D|<|D|$.

If $M$ is an HS D-module, then conditions (a)-(d) hold. Conversely, if conditions (a), (b), and $(d)$ hold, then $M$ is an HS D-module.

Corollary 43. Suppose that $D$ is an uncountable principal ideal domain with exactly $n$ maximal ideals $J_{1}, J_{2}, \ldots, J_{n}$. Suppose further that $\left|D / J_{i}\right|<|D|$ for $1 \leq i \leq n$ (such domains exist by Theorem 2.3 of [33]). If $K$ is the quotient field of $D$, then the HS modules over $D$ are precisely (up to D-module isomorphism) the D-submodules of $K$ containing $D$.

\section{A Generalization of Kaplansky's Problem}

We begin this section by giving the canonical solution to a well-known problem of Kaplansky [27].

Show that if $G$ is an infinite Abelian group with the property that $G / H$ is finite for all nonzero subgroups $H$ of $G$, then $G \cong \mathbb{Z}$.

Solution 1. Assume that $G$ is as stated. Let $g \in G$ be nonzero. It is easy to see that $G=(g, X)$, where $X$ is a complete set of coset representatives for $G$ modulo $(g)$. Since $G /(g)$ is finite, it follows that $G$ is finitely generated. Thus, by the fundamental theorem of finitely generated Abelian groups, $G$ is a finite direct sum of cyclic groups. Since $G$ is infinite, at least one summand must be isomorphic to $\mathbb{Z}$. There can be no other summands; let $\mathbb{Z}$ be an infinite proper homomorphic image of $G$. Thus $G \cong \mathbb{Z}$, and the solution is complete. 
The purpose of this section is to generalize Kaplansky's problem to modules over an arbitrary commutative ring. To do this, we will need the following two lemmas.

Lemma 44 (see [36, (iii) of Lemma 3.1]). Let $R$ be a ring, and suppose that $M$ is an HS R-module. If $N$ is a nonzero submodule of $M$, then $N$ is also an HS R-module.

Proof. We assume that $R$ is a ring, $M$ is an HS $R$-module, and that $N$ is a nonzero submodule of $M$. Since $M$ is HS, $|M / N|<$ $|M|$. However, we also have $|M / N| \cdot|N|=|M|$. Since $M$ is infinite, we deduce that $|N|=|M|$. Now let $K$ be a nonzero submodule of $N$. We will prove that $|N / K|<|N|$. Toward this end, notice that $K$ is a nonzero submodule of $M$. As $M$ is HS, we conclude that $|M / K|<|M|$. Lastly, note that $N / K$ is a submodule of $M / K$, whence $|N / K| \leq|M / K|<|M|=|N|$, and the proof is complete.

Lemma 45 (see [30, Corollary 2.4]). Let $R$ be a commutative ring. Then every nonzero ideal $I$ of $R$ is of finite index in $R$ if and only if every nonzero prime ideal of $R$ is finitely generated and of finite index in $R$.

Before stating our generalization, we remind the reader that (as per our comments following Proposition 41) we may restrict to faithful modules over a domain which is not a field without loss of generality. We now generalize Kaplansky's problem to modules over an arbitrary commutative ring.

Theorem 46 (see [36, Theorem 4.2]). Let $D$ be a domain (which is not a field) with quotient field $K$ and let $M$ be an infinite, faithful $D$-module. Then $M / N$ is finite for every nonzero submodule $N$ of $M$ (i.e., $M$ is HF) if and only if the following hold.

(a) $D$ is a one-dimensional Noetherian domain with all residue fields finite.

(b) $D \subseteq M \subseteq K$ (up to D-module isomorphism).

(c) $M$ is finitely generated over $D$.

Proof. Let $D$ be a domain (which is not a field) with quotient field $K$ and let $M$ be an infinite, faithful module over $D$. Assume first that $M$ is HF. Theorem 42 implies that (b) holds. The proof of Lemma 44 and (b) imply that $D$ is $\mathrm{HF}$ as a module over itself. We now invoke Lemma 45 and Cohen's theorem to conclude that $D$ is Noetherian with all residue fields finite. If $P$ is any nonzero prime ideal of $D$, then as $D / P$ is finite; it follows that $D / P$ is a field. Hence $P$ is a maximal ideal. Thus $D$ is one-dimensional and (a) holds. We now prove that (c) holds as follows. Let $m \in M$ be nonzero. Then $M /(m)$ is finite. If $S$ is a complete set of coset representatives for $M$ modulo $(m)$, then $M=(S, m)$. Hence $M$ is finitely generated.

Conversely, suppose that conditions (a)-(c) hold. It follows from Lemma 45 that $D$ is HF as a module over itself. Since $M$ is finitely generated and $D \subseteq M \subseteq K$, we deduce that there exists some nonzero $d \in D$ such that $d M \subseteq D$. But note that $M \cong_{D} d M$, and thus $M$ is isomorphic to a submodule (ideal) of $D$. It now follows from Lemma 44 that $M$ is HF over D.

\section{Strongly HS Modules}

We begin this section by noting that if $M$ is an HS module over the ring $R$, then $|M / N| \neq|M|$ for every nonzero $R$ submodule $N$ of $M$. We now strengthen the definition of "HS module" as follows.

Definition 47. Let $R$ be a ring and let $M$ be an $R$-module (unlike the definition of "HS module," we allow $M$ to be finite). Then we call $M$ strongly HS provided that $|M / K| \neq|M / N|$ for any two distinct $R$-submodules $K$ and $N$ of $M$.

The terminology is justified since an infinite strongly HS $R$-module $M$ is clearly also an HS $R$-module. As a precursor to our work, we classify the strongly HS Abelian groups.

Proposition 48. Let $G$ be an Abelian group. Then $G$ is strongly $H S$ (i.e., $G$ is a strongly HS $\mathbb{Z}$-module) if and only if $G \cong \mathbb{Z} /(n)$ for some positive integer $n$ or $G \cong \mathbb{Z}$.

Proof. Consider first the group $\mathbb{Z} /(n)$, where $n$ is a positive integer. Proposition 29 implies that $\mathbb{Z} /(n)$ is strongly Jónsson. Hence distinct subgroups of $\mathbb{Z} /(n)$ have distinct cardinalities. Since $\mathbb{Z} /(n)$ is finite, we deduce that distinct factor groups of $\mathbb{Z} /(n)$ have distinct cardinalities, whence $\mathbb{Z} /(n)$ is strongly HS. It is clear that $\mathbb{Z}$ is a strongly HS Abelian group. Conversely, suppose that $G$ is an arbitrary strongly HS Abelian group. If $G$ is finite, then $G$ is also strongly Jónsson, whence, by Proposition $29, G \cong \mathbb{Z} /(n)$ for some positive integer $n$. Now suppose that $G$ is infinite. We claim that $G$ is countable. If $G$ is uncountable, then simply choose an arbitrary countably infinite subgroup $H$ of $G$. Then $|G / H|=|G|=|G /\{0\}|$, contradicting that $G$ is strongly HS. Thus $G$ is countable. But then $G / H$ is finite for every nonzero subgroup $H$ of $G$. We conclude via Kaplansky's problem that $G \cong \mathbb{Z}$, and the proof is complete.

Analogous to Section 6, we now turn our attention toward describing the strongly HS modules over an arbitrary ring. Note first that if $R$ is a ring and $M$ is a finite $R$-module, then $M$ is strongly HS if and only if $M$ is strongly Jónsson. Since we have already characterized the finite strongly Jónsson $R$ modules (Theorem 36), it suffices to consider only infinite strongly HS modules. Since every infinite strongly HS module is HS, it suffices by the comments following Proposition 41 to restrict our study even further to infinite, faithful strongly HS modules over a domain $D$ which is not a field. We now present a classification of the strongly HS modules.

Theorem 49 (see [37, Theorem 2]). Let D be a domain which is not a field, and let $M$ be an infinite faithful D-module. Then $M$ is a strongly HS D-module if and only if the following hold:

(a) D is a Dedekind domain with all residue fields finite,

(b) If $P$ and $Q$ are distinct maximal ideals of $D$, then $D / P$ and $D / Q$ have distinct (nonzero) characteristics,

(c) $M \cong_{D} I$ for some nonzero ideal I of $D$. 
In view of the previous theorem, it suffices to restrict our study of strongly HS modules to domains which are strongly HS as modules over themselves. Let us agree to call a domain $D$ a strongly $H S$ domain if and only if $D$ is strongly HS as a module over itself (i.e., if $I \neq J$ are ideals of $D$, then $|D / I| \neq|D / J|)$.

Corollary 50 (see [37, Corollary 4]). Let D be a domain which is not a field.

(a) Suppose that $D$ has prime characteristic $p$. Then $D$ is a strongly HS domain if and only if $D$ is a DVR with a finite residue field.

(b) Suppose that $D$ has characteristic 0 (and hence $\mathbb{Z} \subseteq$ $D$ ). Then $D$ is a strongly HS domain if and only if $D$ is a Dedekind domain with all residue fields finite and with the additional property that the map $P \mapsto P \cap \mathbb{Z}$ is an injection from $\operatorname{Max}(D)$ into $\operatorname{Max}(\mathbb{Z})$.

(c) If $D$ is a strongly HS domain, then $|D| \leq 2^{\aleph_{0}}$.

Proof. Let $D$ be a domain which is not a field.

(a) Suppose first that $D$ has characteristic $p$. If $D$ is a DVR with a finite residue field, then it follows immediately from the previous theorem that $D$ is a strongly HS domain. Conversely, suppose that $D$ is a strongly HS domain. Let $J$ be an arbitrary maximal ideal of $D$. Since $D$ has characteristic $p$, clearly so does $D / J$. Theorem 49 implies that $J$ is the unique maximal ideal of $D$. Thus $D$ is a local Dedekind domain, whence a DVR. That $D$ has a finite residue field now follows from (a) of Theorem 49.

(b) This follows easily from (b) of Theorem 49.

(c) Assume now that $D$ is a strongly HS domain. Then $D$ is Dedekind, whence Noetherian. Let $P$ be a maximal ideal of $D$. Krull's intersection theorem yields that $\bigcap_{i=1}^{\infty} P^{i}=\{0\}$. It follows that $D$ maps injectively into $\prod_{i=1}^{\infty} D / P^{i}$. But since $D$ is a residually finite Dedekind domain, it is easy to see by induction that $D / P^{n}$ is finite for every positive integer $n$. Thus $|D| \leq$ $\left|\prod_{i=1}^{\infty} D / P^{i}\right| \leq\left|\prod_{i=1}^{\infty} \mathbb{N}\right|=\aleph_{0}^{\aleph_{0}}=2^{\aleph_{0}}$.

We now prove that for every prime number $p$ and every cardinal number $\kappa$ satisfying $\aleph_{0} \leq \kappa \leq 2^{\aleph_{0}}$, there is a strongly HS domain of characteristic $p$ and cardinality $\kappa$.

Example 51. Let $p$ be a prime and let $\kappa$ be a cardinal number satisfying $\aleph_{0} \leq \kappa \leq 2^{\aleph_{0}}$. There exists a strongly HS domain (which is not a field) of characteristic $p$ and of cardinality $\kappa$.

Proof. Let $F:=\mathbb{F}_{p}$ be the field of $p$ elements, let $F[t]$ be the polynomial ring over $F$ in the variable $t$, and let $F[[t]]$ be the ring of formal power series over $F$ in the variable $t$. The underlying set of $F[[t]]$ is the set of all functions from $\mathbb{N}$ into $F$, whence $|F[[t]]|=2^{\aleph_{0}}$. The quotient field of $F[t]$ is the field $F(t)$ of rational functions in $t$; the quotient field of $F[[t]]$ is the field $F((t))$ of formal Laurent series in $t$. There is a field $K$ of cardinality $\kappa$ such that $F(t) \subseteq K \subseteq F((t))$. Note that $F[[t]]$ is a DVR on $F((t))$ (i.e., $F[[t]]$ is a DVR with quotient field $F((t)))$, $K \subseteq F((t))$, and $F[[t]] \cap K$ is not a field (since $t$ is not invertible in $F[[t]]$ ). It follows that $F[[t]] \cap K$ is a DVR on $K$ (whence also has cardinality $\kappa)$ with maximal ideal $M:=(t) \cap K$. It is obvious that $F[[t]] \cap K$ has characteristic $p$. It is also easy to check that $F$ maps injectively into $(F[[t]] \cap K) / M$ and $(F[[t]] \cap K) / M$ maps injectively into $F[[t]] /(t) \cong F$. It follows that $|(F[[t]] \cap K) / M|=|F|=p$. We have shown that $F[[t]] \cap K$ is a DVR of characteristic $p$ and of cardinality $\kappa$ and that $F[[t]] \cap K$ has residue field isomorphic to $F$, which is finite. Part (a) of Corollary 50 yields that $F[[t]] \cap K$ is a strongly HS domain, and the proof is complete.

Given the previous example, it is natural to enquire about the characteristic 0 case. Before giving a more general result, we remark that the ring $J_{p}$ of $p$-adic integers is a DVR of characteristic 0 (of cardinality $2^{\aleph_{0}}$ ) with residue field isomorphic to $\mathbb{Z} /(p)$, whence $J_{p}$ is a strongly HS domain by (b) of Corollary 50.

Example 52. Let $\kappa$ be a cardinal number satisfying $\aleph_{0} \leq$ $\kappa \leq 2^{\aleph_{0}}$. Further, let $p_{1}, p_{2}, \ldots, p_{n}$ be distinct primes and let $k_{1}, k_{2}, \ldots, k_{n}$ be positive integers. There exists a principal ideal domain $D$ of cardinality $\kappa$ with exactly $n$ maximal ideals $M_{1}, M_{2}, \ldots, M_{n}$ with the property that $D / M_{i} \cong \mathbb{F}_{p_{i}^{k_{i}}}$ for each $i, 1 \leq i \leq n$. Hence $D$ is a strongly HS domain.

Proof. The existence of such a $D$ with the above properties is established in Theorem 2.6 of [32]. The construction is quite technical, and we suppress the details here (we remark that the ideas of the construction are due to C. Shah; see Theorem 2.3 of [33]). We deduce immediately from (b) of Theorem 49 that $D$ is a strongly HS domain.

We conclude this section with two propositions. The first shows that the strongly HS property behaves badly with respect to integral extensions; the second demonstrates that the property behaves as nicely as possible with respect to overrings.

Proposition 53 (see [37, Proposition 11]). Let D be a strongly HS domain, and suppose that $R$ is a finite integral extension of $D$ (i.e., $R$ is integral over $D$ and has a finite basis as a $D$ module). Then $R$ need not be strongly HS.

Proof. Let $\mathbb{Z}[i]:=\{a+b i: a, b \in \mathbb{Z}\}$ be the ring of Gaussian integers. Then clearly $\mathbb{Z}[i]$ is integral over $\mathbb{Z}$ and $\{1, i\}$ forms a $\mathbb{Z}$-basis for $\mathbb{Z}[i]$. Recall that the function $N: \mathbb{Z}[i] \rightarrow \mathbb{N}$ given by $N(a+b i):=a^{2}+b^{2}$ is a Euclidean norm (from which one proves that $\mathbb{Z}[i]$ is a Euclidean domain). Consider the ideals $(1+2 i)$ and $(1-2 i)$ of $\mathbb{Z}[i]$. Since $N(1+2 i)=N(1-2 i)=5$, which is prime, we conclude that $1+2 i$ and $1-2 i$ are Gaussian primes. Thus $(1+2 i)$ and $(1-2 i)$ are maximal ideals of $\mathbb{Z}[i]$. The units of $\mathbb{Z}[i]$ are precisely the elements of $\mathbb{Z}[i]$ which have norm 1 . It follows (and is well known, of course) that the units of $\mathbb{Z}[i]$ are exactly $1,-1, i$, and $-i$. From this fact, it is clear that $1+2 i$ and $1-2 i$ are not associates, whence $(1+2 i)$ and $(1-2 i)$ 
are distinct maximal ideals of $\mathbb{Z}[i]$. Note that $5 \in(1+2 i) \cap$ $(1-2 i)$, whence $(1+2 i) \cap \mathbb{Z}=(1-2 i) \cap \mathbb{Z}=5 \mathbb{Z}$. Corollary 50 part (b) implies that $\mathbb{Z}[i]$ is not a strongly HS domain.

Proposition 54 (see [37, Theorem 3]). Let D be a strongly HS domain. Then every overring of $D$ is a strongly HS domain.

\section{Independence}

Given the set-theoretic nature of our results, it is natural to ask the following question: are there "natural" statements about Jónsson and HS modules which are independent of ZFC? In other words, are there Jónsson and HS-theoretic statements which can neither be proved nor refuted from the axioms of ZFC (assuming ZFC is consistent)? The answer is "yes," and the purpose of this section is to give a sampling of two such statements.

Before doing so, we will need some additional terminology. Let $R$ be a ring, and let $M$ be an infinite $R$-module. Following the literature, $M$ is said to be congruent provided $N \cong_{R} M$ for every submodule $N$ of $M$ of the same cardinality as $M$. Note trivially that every Jónsson $R$-module is congruent. We will make use of the following lemma.

Lemma 55 (see [44, Theorem 2]). Assume that GCH holds. Now let $D$ be an arbitrary uncountable Noetherian domain and suppose that $|D|$ is not the successor of a cardinal of countable cofinality. If $M$ is a faithful congruent module over $D$, then $M$ is free and $D$ is a Dedekind domain.

We now present our first independence result.

Proposition 56. Let $a>0$ be an ordinal, and suppose that the cardinal $\aleph_{a}$ is not the successor of a cardinal of countable cofinality. Then it is undecidable in ZFC whether there exists a Noetherian domain D of size $\aleph_{a}$ which is not Dedekind and which admits a faithful Jónsson module.

Proof. Let $a>0$ be an ordinal, and assume that $\aleph_{a}$ is not the successor of a cardinal of countable cofinality.

Assume first that GCH holds. Now suppose that $D$ is a Noetherian domain of size $\aleph_{a}$ which admits a faithful Jónsson module. Then by Lemma $55, D$ is Dedekind. Hence $\mathrm{ZFC}+\mathrm{GCH}$ proves that "there does not exist a Noetherian domain $D$ of size $\aleph_{a}$ which is not Dedekind and which admits a faithful Jónsson module."

Now suppose that $\mathrm{CH}$ (the continuum hypothesis) fails. It is known (see Chapter 7 of Kunen [45], e.g.) that it is consistent with ZFC that CH can fail so badly as to have $\aleph_{a} \leq$ $2^{\aleph_{0}}$. So suppose this is the case, and consider the quasicyclic group $\mathbb{Z}\left(p^{\infty}\right)$. It is well known that the endomorphism ring of $\mathbb{Z}\left(p^{\infty}\right)$ (as a $\mathbb{Z}$-module) is the DVR $J_{p}$ of $p$-adic integers. It is also well known that $J_{p}$ has characteristic 0 and that $\left|J_{p}\right|=$ $2^{\aleph_{0}}$. Now, $\mathbb{Z}\left(p^{\infty}\right)$ is naturally a faithful Jónsson module over $J_{p}$, whence (as $\mathbb{Z}\left(p^{\infty}\right)$ is a Jónsson Abelian group) a faithful Jónsson module over every subring $R$ of $J_{p}$. Let $\left\{t_{i}: i<\right.$ $\left.\aleph_{a}\right\} \cup\{x\} \subseteq J_{p}$ be algebraically independent over $\mathbb{Z} \subseteq J_{p}$, and let $K$ be the quotient field of $\mathbb{Z}\left[t_{i}: i<\aleph_{a}\right]$. Then $D:=$ $K \cap J_{p}$ is a DVR on $K$ whence has cardinality $\aleph_{a}$. Observe that $D[x] \subseteq J_{p}$ is Noetherian and two-dimensional, whence not Dedekind, but has cardinality $\aleph_{a}$ and admits the faithful Jónsson module $\mathbb{Z}\left(p^{\infty}\right)$. Hence ZFC $+\aleph_{a} \leq 2^{\aleph_{0}}$ proves that "there exists a Noetherian domain $D$ of size $\aleph_{a}$ which is not Dedekind and which admits a faithful Jónsson module."

We now present a similar HS-theoretic independence result. We begin with two lemmas and then conclude the section with a proposition.

Lemma 57 (see [4, (c) of Theorem 5.15]). Suppose that GCH holds, and let $\kappa$ and $\lambda$ be infinite cardinals. If $\lambda<c f(\kappa)$, then $\kappa^{\lambda}=\kappa$.

Lemma 58 (see [32, Lemma 2.1]). Let $D$ be a Noetherian domain, that is, not a finite field, and let $I$ be a proper ideal of D. If $|D|=\rho$ and $|D / I|=\kappa$, then $\kappa+\aleph_{0} \leq \rho \leq \kappa^{\aleph_{0}}$.

Proposition 59. Let $a>0$ be an ordinal, and suppose that the cardinal $\aleph_{a}$ is not the successor of a cardinal of countable cofinality. Then it is undecidable in ZFC whether there exists a Noetherian domain D of size $\aleph_{a}$ which is not a field and which admits a faithful HS module.

Proof. Let $a>0$ be an ordinal, and suppose that $\aleph_{a}$ is not the successor of a cardinal of countable cofinality.

As above, we first assume that GCH holds. We will show that there does not exist a Noetherian domain $D$ of size $\aleph_{a}$ which is not a field and which admits a faithful HS module. Suppose by way of contradiction that such a domain $D$ exists. Then by Theorem $42, D$ is HS as a module over itself. Since $D$ is not a field, there exists a proper nonzero ideal $I$ of $D$. Let $|D / I|:=\kappa$. Lemma 58 along with the fact that $D$ is HS over itself yields

$$
\kappa<\aleph_{a} \leq \kappa^{\aleph_{0}} .
$$

We first claim that $\kappa$ is infinite. For if $\kappa$ were finite, then (8) along with the fact that $a>0$ yields that $\aleph_{1} \leq \aleph_{a} \leq \kappa^{\aleph_{0}}=$ $2^{\aleph_{0}}=\aleph_{1}$, whence $\aleph_{a}=\aleph_{1}$. However, $\aleph_{1}$ is the successor of $\aleph_{0}$, and $\aleph_{0}$ has countable cofinality. This contradicts the fact that $\aleph_{a}$ is not the successor of a cardinal of countable cofinality. Thus $\kappa$ is infinite. We again use (8) to get $\kappa<\aleph_{a} \leq \kappa^{\aleph_{0}} \leq$ $\kappa^{\kappa}=2^{\kappa}=\kappa^{+}$. We conclude that $\aleph_{a}=\kappa^{+}$. Invoking (8) yet again, we conclude that

$$
\aleph_{a}=\kappa^{+} \leq \kappa^{\aleph_{0}} .
$$

Since $\aleph_{a}$ is not the successor of a cardinal of countable cofinality, we conclude from (9) above that $\aleph_{0}<c f(\kappa)$. But then by Lemma 57, we deduce that $\kappa^{\aleph_{0}}=\kappa$, and this contradicts (9). We have shown that $\mathrm{ZFC}+\mathrm{GCH}$ proves that "there does not exist a Noetherian domain $D$ of size $\aleph_{a}$ which is not a field and which admits a faithful HS module."

As in the proof of Proposition 56, we now assume that $\aleph_{a} \leq 2^{\aleph_{0}}$. In this case, Example 51 yields a Noetherian strongly HS domain $D$ of size $\aleph_{a}$ which is not a field. Thus ZFC $+\aleph_{a} \leq 2^{\aleph_{0}}$ proves that "there exists a Noetherian domain $D$ of size $\aleph_{a}$ which is not a field and which admits a faithful HS module." 


\section{Open Problems}

We conclude this survey with several open problems for further research.

Open Problem 1. The countably infinite Jónsson modules have been classified. Can one classify the uncountable Jónsson modules over an arbitrary ring (or over some restricted class of rings, such as valuation rings)?

Open Problem 2. ZFC $+\mathrm{GCH}$ proves the nonexistence of large Jónsson modules. Can the nonexistence of large Jónsson modules be proved in $\mathrm{ZFC}$ alone?

Open Problem 3. Let $D$ be a Noetherian domain which is not a field. ZFC + GCH proves that every faithful Jónsson $D$-module is countable. Is ZFC sufficient to prove that every faithful Jónsson $D$-module is countable?

Open Problem 4. Recall that a Jónsson ring is a ring for which every proper subring has smaller cardinality. It is known that every uncountable Jónsson ring is a noncommutative division ring. Do such rings exist?

\section{Conflict of Interests}

The author declares that there is no conflict of interests regarding the publication of this paper.

\section{References}

[1] P. Erdős and A. Hajnal, "On a problem of B. Jónsson," Bulletin de l'Académie Polonaise des Sciences, vol. 14, pp. 19-23, 1966.

[2] F. Rowbottom, Some strong axioms of infinity incompatible with the axiom of constructibility [Ph.D. thesis], University of Wisconsin, Madison, Wis, USA, 1964.

[3] J. Silver, Some applications of model theory in set theory [Ph.D. thesis], University of California, Berkeley, Calif, USA, 1966.

[4] T. Jech, Set Theory, Springer Monographs in Mathematics, Springer, Berlin, Germany, 2003.

[5] E. Coleman, "Jonsson groups, rings and algebras," Irish Mathematical Society Bulletin, no. 36, pp. 34-45, 1996.

[6] L. Fuchs, Infinite Abelian Groups. Vol. I, vol. 36 of Pure and Applied Mathematics, Academic Press, New York, NY, USA, 1970.

[7] W. R. Scott, "Groups and cardinal numbers," American Journal of Mathematics, vol. 74, pp. 187-197, 1952.

[8] A. Ju. Olšanskiǔ, "An infinite group with subgroups of prime orders," Izvestiya Akademii Nauk SSSR. Seriya Matematicheskaya, vol. 44, no. 2, pp. 309-321, 1980.

[9] S. Shelah, "On a problem of Kurosh, Jónsson groups, and applications," in Word Problems II (Proc. Conf. Oxford, 1976), Studies in Logic and the Foundations of Mathematics, pp. 373394, North-Holland, Amsterdam, The Netherlands, 1980.

[10] R. McKenzie, "On semigroups whose proper subsemigroups have lesser power," Algebra Universalis, vol. 1, no. 1, pp. 21-25, 1971.

[11] T. J. Laffey, "Infinite rings with all proper subrings finite," The American Mathematical Monthly, vol. 81, pp. 270-272, 1974.
[12] A. Kostinsky, Some problems for rings and lattices within the domain of general algebra [Ph.D. thesis], University of California, Berkeley, Calif, USA, 1969.

[13] R. Gilmer and W. Heinzer, "On Jónsson modules over a commutative ring," Acta Scientiarum Mathematicarum, vol. 46, no. 1-4, pp. 3-15, 1983.

[14] R. Gilmer and W. Heinzer, "An application of Jónsson modules to some questions concerning proper subrings," Mathematica Scandinavica, vol. 70, no. 1, pp. 34-42, 1992.

[15] R. Gilmer and W. Heinzer, "Cardinality of generating sets for modules over a commutative ring," Mathematica Scandinavica, vol. 52, no. 1, pp. 41-57, 1983.

[16] R. Gilmer and W. Heinzer, "Jónsson $\omega_{0}$-generated algebraic field extensions," Pacific Journal of Mathematics, vol. 128, no. 1, pp. 81-116, 1987.

[17] R. Gilmer and W. Heinzer, "On the cardinality of subrings of a commutative ring," Canadian Mathematical Bulletin, vol. 29, no. 1, pp. 102-108, 1986.

[18] R. Gilmer and W. Heinzer, "On Jónsson algebras over a commutative ring," Journal of Pure and Applied Algebra, vol. 49, no. 1-2, pp. 133-159, 1987.

[19] R. G. Burns, F. Okoh, H. Smith, and J. Wiegold, "On the number of normal subgroups of an uncountable soluble group," Archiv der Mathematik, vol. 42, no. 4, pp. 289-295, 1984.

[20] A. Ecker, "The number of submodules," in Abelian Groups and Modules (Dublin, 1998), Trends in Mathematics, pp. 359-368, Birkhäuser, Basel, Switzerland, 1999.

[21] W. Heinzer and D. Lantz, "Artinian modules and modules of which all proper submodules are finitely generated," Journal of Algebra, vol. 95, no. 1, pp. 201-216, 1985.

[22] J. Lawrence, "Independent sets, Jonsson modules and the number of submodules," The Journal of the Indian Mathematical Society, vol. 48, no. 1-4, pp. 119-130, 1984.

[23] W. D. Weakley, "Modules whose proper submodules are finitely generated," Journal of Algebra, vol. 84, no. 1, pp. 189-219, 1983.

[24] G. Oman, "Jónsson modules over commutative rings", in Commutative Rings: New Research, pp. 1-6, Nova Science Publishers, New York, NY, USA, 2009.

[25] G. Oman, "Jónsson modules over noetherian rings," Communications in Algebra, vol. 38, no. 9, pp. 3489-3498, 2010.

[26] G. Oman, "Some results on jonsson modules over a commutative ring," Houston Journal of Mathematics, vol. 35, no. 1, pp. $1-12,2009$.

[27] I. Kaplansky, Infinite Abelian Groups, University of Michigan Press, Ann Arbor, Mich, USA, 1954.

[28] B. A. Jensen and D. W. Miller, "Commutative semigroups which are almost finite," Pacific Journal of Mathematics, vol. 27, pp. 533-538, 1968.

[29] R. P. Tucci, "Commutative semigroups whose proper homomorphic images are all of smaller cardinality," Kyungpook Mathematical Journal, vol. 46, no. 2, pp. 231-233, 2006.

[30] K. L. Chew and S. Lawn, "Residually finite rings," Canadian Journal of Mathematics, vol. 22, pp. 92-101, 1970.

[31] K. B. Levitz and J. L. Mott, "Rings with finite norm property," Canadian Journal of Mathematics, vol. 24, pp. 557-565, 1972.

[32] K. A. Kearnes and G. Oman, "Cardinalities of residue fields of noetherian integral domains," Communications in Algebra, vol. 38, no. 10, pp. 3580-3588, 2010.

[33] C. Shah, "One-dimensional semilocal rings with residue domains of prescribed cardinalities," Communications in Algebra, vol. 25, no. 5, pp. 1641-1654, 1997. 
[34] M. Orzech and L. Ribes, "Residual finiteness and the Hopf property in rings," Journal of Algebra, vol. 15, no. 1, pp. 81-88, 1970.

[35] K. Varadarajan, "Residual finiteness in rings and modules," Journal of the Ramanujan Mathematical Society, vol. 8, no. 1-2, pp. 29-48, 1993.

[36] G. Oman and A. Salminen, "On modules whose proper homomorphic images are of smaller cardinality," Canadian Mathematical Bulletin, vol. 55, no. 2, pp. 378-389, 2012.

[37] G. Oman, "Strongly Jónsson and strongly HS modules," Journal of Pure and Applied Algebra. In press.

[38] R. Gilmer, Multiplicative Ideal Theory, vol. 90 of Queen's Papers in Pure and Applied Mathematics, Queen's University, Kingston, Canada, 1992.

[39] K. Gödel, "The consistency of the axiom of choice and of the generalized continuum hypothesis with the axioms of set theory," Uspekhi Matematicheskikh Nauk, vol. 3, no. 1(23), pp. 96149, 1948.

[40] D. D. Anderson, "Artinian modules are countably generated," Colloquium Mathematicum, vol. 38, no. 1, pp. 5-6, 1977.

[41] L. Fuchs, Abelian Groups, Publishing House of the Hungarian Academy of Sciences, Budapest, Hungary, 1958.

[42] T. W. Hungerford, Algebra, vol. 73 of Graduate Texts in Mathematics, Springer, New York, NY, USA, 1980.

[43] R. Baer, "Situation der untergruppen und struktur der gruppe," Sitzungsberichte der Heidelberger Akademie der Wissenschaften, vol. 2, pp. 12-17, 1933.

[44] G. Oman, "More results on congruent modules," Journal of Pure and Applied Algebra, vol. 213, no. 11, pp. 2147-2155, 2009.

[45] K. Kunen, Set Theory. An Introduction to Independence Proofs, vol. 102 of Studies in Logic and the Foundations of Mathematics, North-Holland, Amsterdam, The Netherlands, 1980. 


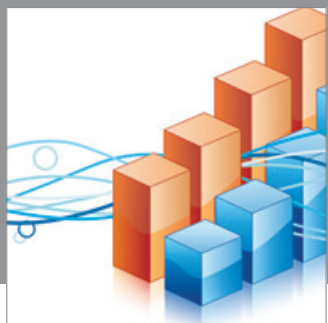

Advances in

Operations Research

mansans

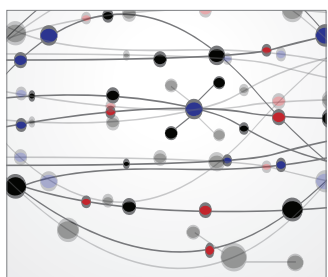

The Scientific World Journal
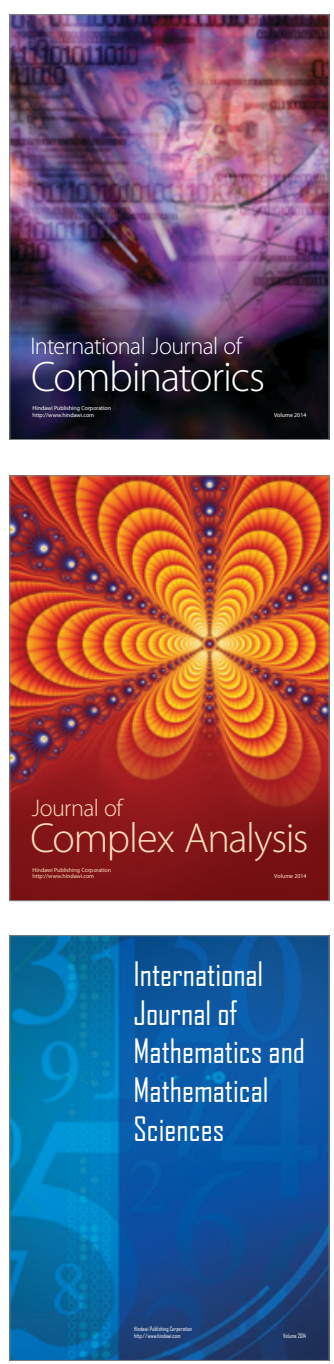
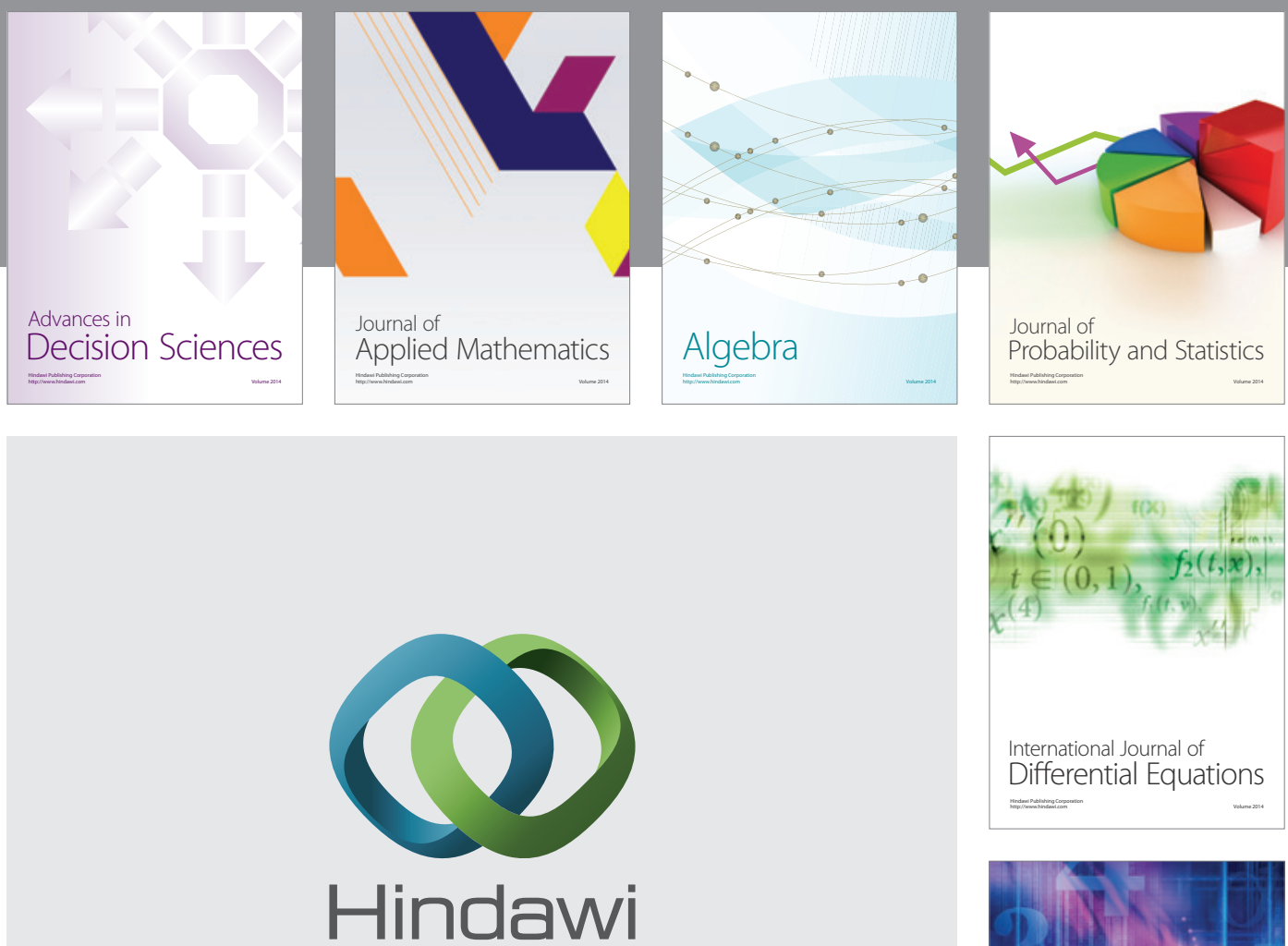

Submit your manuscripts at http://www.hindawi.com
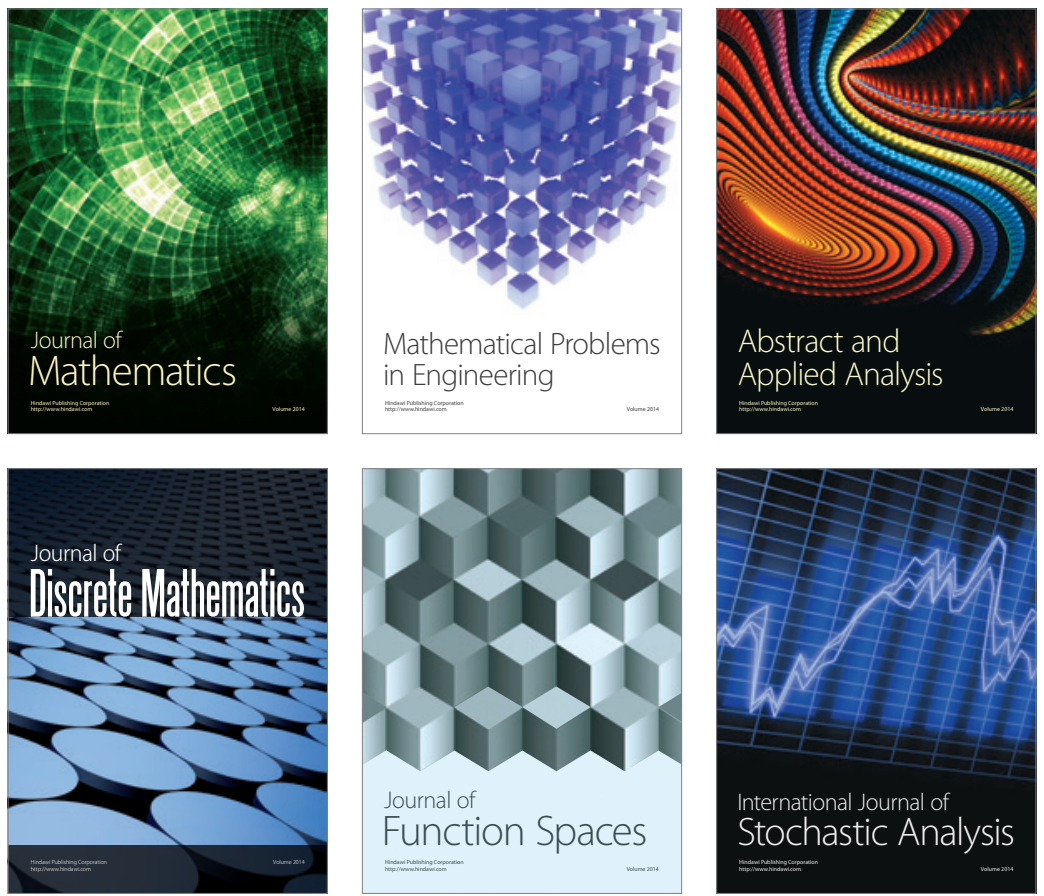

Journal of

Function Spaces

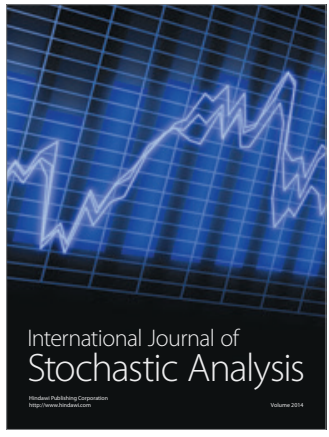

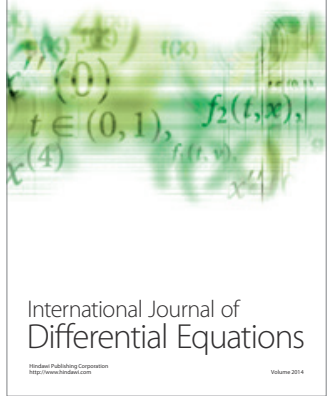
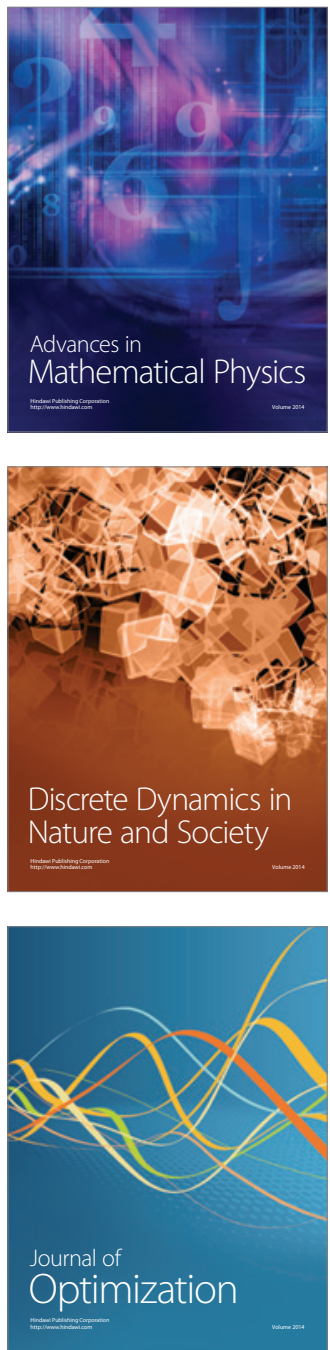